\title{
LA GESTIÓN DEL REGADÍO CATALÁN: ENTRE EFICIENCIA, COMPATIBILIDAD DE USOS Y LEGITIMIDAD SOCIAL
}

\author{
Sandra Ricart y David Pavón \\ Departamento de Geografía \\ Universidad de Girona
}

\section{RESUMEN}

En los años precedentes, el regadío y su gestión han devenido protagonistas de un debate social que cuestiona los límites económicos, ambientales y territoriales de su práctica en espacio y tiempo. Las construcciones hidráulicas como los canales de regadío han ocupado un papel central en el intento de "dominar" el recurso agua y controlar así el territorio. Sin embargo y, con el paso del tiempo, tanto la modernización de regadíos tradicionales como la promoción de nuevos regadíos han sido progresivamente cuestionadas ante el auge de las demandas ambientales y la promoción de la gobernanza como mecanismo favorecedor de acuerdos entre partes interesadas. En Cataluña, la gestión del regadío debe hacer frente tanto a los requisitos de eficiencia como a la compatibilidad entre usos consuntivos y no consuntivos así como a la legitimación social de proyectos que sobrepasan el interés sectorial. El análisis de la realidad asociada a los regadíos históricos del Bajo Ter y de la Muga así como del proyecto en ejecución del canal Segarra-Garrigues enfatiza en la necesidad de promover un modelo de gestión territorial del regadío capaz de integrar y legitimar distintos puntos de vista en competencia.

Palabras clave: gestión del regadío, eficiencia, compatibilidad de usos, competencia, legitimidad social, regadíos del Bajo Ter y de la Muga, canal Segarra-Garrigues

\section{ABSTRACT}

\section{Catalan irrigation management: between efficiency, compatibility of uses and social legitimacy}

In previous years, irrigation and its management have become protagonists of a social debate that questions their economic, environmental and territorial limits in space and time. The hydraulic constructions as irrigation canals have played a central role in the attempt to "dominate" the water resources and so control the territory. However and after some time, both the modernization of traditional irrigation as the promotion of new irrigation projects are called into question due to the rise of environmental demands and promoting governance as a mechanism favourable to agreements between stakeholders. In Catalonia, the irrigation management must deal both efficiency requirements as to the compatibility between consumptive and non-consumptive water uses well as the social legitimacy of projects that exceed sectoral interest. The situation analysis of Bajo Ter and Muga historic irrigation canals and the running project of Segarra-Garrigues irrigation canal emphasize the need to promote a territorial management model capable of integrating and legitimize different competing water views.

Keywords: Irrigation management, efficiency, compatibility of uses, competence, social legitimacy, Bajo Ter and Muga irrigation, Segarra-Garrigues canal

\section{INTRODUCCIÓN}

De la misma manera que ha acontecido en otros sectores de la política pública, el interés por el medio ambiente y la gestión de los recursos hídricos ha ganado relevancia en la dinámica de la agricultura. El regadío y su gestión han devenido así protagonistas de un debate donde parte de la sociedad cuestiona

Contacto: Sandra Ricart: sandra.ricart@udg.edu; David Pavón: david.pavon@udg.edu 
los límites sociales, económicos, ambientales y territoriales de su práctica (Berger y Roques, 2005; Özerol et al., 2012). Si bien muchos de los factores iniciales que impulsaron su desarrollo -alimentación, asentamiento de la población, dinamización de la economía rural- siguen vigentes en la actualidad, los retos a los que debe hacer frente han ido en aumento en las últimas décadas (Burton, 2010). Con ello su gestión ha tendido a la búsqueda del equilibrio necesario entre los condicionantes físicos que impone el cambio climático o las externalidades negativas sobre los ecosistemas, así como el reto de la soberanía alimentaria, la compatibilidad con el resto de usos consuntivos del agua o su legitimación como usuario de bienes comunes (Brebbia et al., 2010; Turral et al., 2010).

Desde la segunda mitad del siglo XIX y a lo largo del siglo XX11, el desarrollo del regadío impulsado a nivel institucional ha emergido como una práctica más en la estrategia política para controlar el territorio², los recursos y la dinámica de la población ${ }^{3}$ (Swyngedouw, 1999). Con ello, las construcciones hidráulicas han ocupado un papel central en el intento de "dominar" el recurso agua intensificando la intervención antrópica sobre el recurso, su estado y los procesos naturales afines (Pardo, 1999). Las llanuras agrícolas han jugado, así, un papel clave en el desarrollo del regadío, donde la presencia de embalses y canales ha articulado la gestión de los recursos naturales y la vertebración de la matriz territorial (Kaika, 2006). En el ámbito mediterráneo, la complejidad en torno a la gestión de los recursos de agua dulce -sobre todo dada su disponibilidad, presión y competencia de uso $^{4}$ - ha centrado el debate político, técnico y social del regadío (Davies y Simonovic, 2011; Wiek y Larson, 2012). A las puertas del siglo XXI y en contextos como el español, la modernización de los regadíos tradicionales y la definición de nuevos proyectos de regadío darán lugar a la combinación de políticas de gestión del agua y de la agricultura. Así lo justificarán autores como Lecina et al. (2010), para quienes ambos procesos responderán al paso de una agricultura subvencionada a una agricultura competitiva así como a su adaptación ante los parámetros ambientales exigidos en las escalas europea y nacional.

\section{LA GESTIÓN DEL REGADÍO EN CATALUÑA: SUMA DE EFICIENCIA Y COMPATIBILIDAD DE USOS}

La relevancia que tiene el regadío en Cataluña por sus implicaciones a nivel territorial, económico, social, de paisaje, etc. resulta innegable. Según el Plan de Regadíos de Cataluña (2008), el 26,26\% de la superficie agraria útil (SAU) es de regadío, lo que equivale a 306.292 ha. De ellas, el 53,7\% se sitúa en la provincia de Lleida seguida, a bastante distancia, por el resto de demarcaciones: Tarragona (26,3\%), Girona (14,2\%) y Barcelona (5,8\%). La cuenca hidrográfica del Ebro en Cataluña, que incluye la totalidad de los regadíos de Lleida y del curso inferior del Ebro, supone alrededor del 50\% del territorio, aunque

1 Ortega Santos (2012) considera los siglos XIX y XX como el periodo en el que tiene lugar el salto cuantitativo -extensión superficial- y cualitativo -regulación de las aguas mediante la gran hidráulica- en la explotación del agua como recurso natural de primer orden. Una idea paralela al hecho según el cual hasta el siglo XIX el agua no será objeto público, momento en que la política hidráulica devendrá una política pública de primer orden (Muñiz, 2009).

2 Según ponen de manifiesto Pervanchon y Blouet (2002), la agricultura de regadío juega un papel clave en el desarrollo del territorio y así lo demuestra el uso del concepto de "agricultura territorial", entendida como una voluntad de situar al agricultor en el centro de su proyecto de riego y de su territorio así como dotarlo de responsabilidades dentro de la colectividad y de la sociedad como un gestor del territorio capaz de luchar contra la desertificación rural.

3 Una dinámica focalizada en distintas partes del mundo occidental como el oeste de los Estados Unidos -con California como caso paradigmático- o países como España, Francia o Italia en su condición de máximos exponentes del desarrollo hidráulico vinculado a la capacidad de colonización (Ertsen, 2006). Para España, López-Gunn (2009) describirá cómo la construcción de hasta 800 presas -y sistemas de riego adyacentes- durante el régimen franquista será una forma de legitimar el poder político a partir del convencimiento de las élites rurales. Ya en 1843 y para Francia, el ingeniero Nadault de Buffon propondrá que sea el Estado quien asuma la carga financiera de la construcción de redes de canales para el riego como mecanismo para asentar la actividad rural en los territorios más pobres del sur del país (Pritchard, 2004). En Italia, la dinámica del regadío se adaptará a una importante intervención pública llevada a cabo bajo un conglomerado de objetivos políticos y socioeconómicos que tendrán en la recuperación de tierras aptas para la actividad agrícola su principal objetivo (Bartolini et al., 2007).

4 En la Europa mediterránea, la agricultura de regadío representa en torno al $60 \%$ de las extracciones totales de agua dulce, si bien su producción está condicionada tanto por la variabilidad física del propio recurso como por la competencia entre usos, especialmente intensa en época de estiaje (Knox et al., 2012). Así, a medida que el agua deviene protagonista según su disponibilidad, la competencia sobre el recurso se intensifica y repercute en su valor, una dinámica que condiciona la adaptación de la agricultura de regadío hacia la priorización de un uso más productivo del agua. Ambos conceptos, disponibilidad y competencia, están vinculados a la "seguridad hídrica", concepto referenciado en la década de los noventa que integra el acceso al agua para el desarrollo de las necesidades humanas así como el mantenimiento de los requisitos ambientales y que se asociará al de "seguridad alimentaria", donde entra en juego el papel del regadío (Cook y Bakker, 2012). 
en él habita menos del 10\% de la población catalana. No obstante, representa el 70\% de la superficie de regadío de toda la región y el $60 \%$ de la demanda anual de agua; de ella, el $95 \%$ se dedica a usos agroganaderos (superior al 70\% para el conjunto de Cataluña).

En 1918, los datos de la Junta Consultiva Agronómica ofrecían un total de 180.532 ha de regadío para Cataluña. Por tanto, la progresión de las cifras atestigua la notable expansión del regadío en la región a lo largo del siglo XX. Esta transformación ha permitido incrementar tanto las producciones como las rentas agrarias y ha sido un factor clave para explicar los cambios en los usos del suelo agrícola y en la mejora de sus rendimientos. Sin la irrigación difícilmente se entenderían los procesos de modernización por los que ha transitado el sector agrario. No obstante, su intensificación también ha contribuido a agravar problemas como la salinización o la eutrofización. A ello se suma la alteración de diversos ecosistemas por la ejecución de infraestructuras hidráulicas a gran escala. Hoy día la compatibilización de los usos hídricos sean estos agrícolas, industriales, urbanos, energéticos o ambientales se presenta como el reto fundamental en la gestión del recurso agua. A tal efecto, el aumento en la eficiencia del agua de riego se revela no como condición suficiente, pero sí necesaria, para que el resultado sea exitoso. En este ámbito se perfilan tres líneas de actuación posibles. En primer lugar, mejorar la eficiencia en la aplicación del agua a las parcelas por parte de los regantes y en las infraestructuras y modalidades organizativas utilizadas para el transporte y distribución del agua de las comunidades de regantes. En segundo lugar, existe la posibilidad de intervenir sobre los precios del agua, para incentivar el ahorro y calibrar los efectos socioeconómicos (Berbel et al., 2009). En tercer lugar se añade la posibilidad de actuar sobre la estructura productiva agrícola de cada área de irrigación (Oca, 2009). Todo ello queda condicionado por las implicaciones de las políticas agrarias y, muy especialmente, por las prioridades marcadas por la Unión Europea.

\subsection{El Plan de Regadíos de Cataluña (2008-2020): entre la modernización y los nuevos proyectos de regadío}

Actualmente y, hasta el año 2020, el documento que establece las orientaciones en materia de regadíos del Principado es el Plan de Regadíos 2008-2020. Aunque el Plan no se deriva de la aplicación de un marco normativo específico que determine la necesidad de su redacción, se creyó conveniente para fijar los ejes de desarrollo de dicho ámbito. El plan propone una expansión notable del área irrigada, pasando de las poco más de 300.000 ha. de 2008 a las 439.016 ha. de 2020 -incluyendo aquí la extensión que sólo contaría con riego de apoyo. Pese a esta dinámica, no habría un incremento en la dotación hídrica final, ya que se produciría una compensación por el ahorro producto de la mejora del riego tradicional. Se confía liberar unos $500 \mathrm{hm}^{3}$ que permitan atender los nuevos regadíos.

Aunque la propuesta asume algunos de les principios de la Nueva Cultura del Agua -modernización de los sistemas de riego tradicionales o utilización de aguas regeneradas-, en cambio se pone excesivo énfasis en la ampliación del regadío y, muy poco, en si el resto de consumos quedan garantizados o si alguno de estos se podrían considerar prioritarios respecto el consumo agrícola. En el informe emitido por el Consell Assessor pel Desenvolupament Sostenible de la Generalitat de Cataluña, en julio de 2010 (CADS, 2010), se cuestionan abiertamente algunas de las pretensiones del Plan. Se subrayan las discrepancias entre algunas de las cifras recogidas en él, en relación con las que dispone la Agencia Catalana del Agua (ACA), básicamente por la posible exacerbación respecto los consumos actuales. Igualmente, expresa que los caudales recuperados por la modernización de los regadíos sería preferible destinarlos a aumentar la garantía de los regadíos actuales y a los requerimientos ambientales de la demarcación hidrográfica. El informe citado muestra su inquietud respecto el modelo social agrario que podría consolidarse con las propuestas de gran regadío, así como una visión que todavía se deja arrastrar por costosas infraestructuras hidráulicas. Cuestiones como la viabilidad de estas ejecuciones o los costes para los regantes y para la Administración deberían haber sido analizadas más sólidamente por el plan, a juicio del CADS. En la misma línea de lo anterior, el informe manifiesta el poco énfasis puesto en las medidas para alcanzar el buen estado ecológico de las masas de agua, sean superficiales o subterráneas. El apartado económico, con una inversión prevista de 4.596 millones de euros, se consideraba poco realista, 
tanto desde la perspectiva de los esfuerzos de financiación que exigía a los regantes como a la propia administración (Garrabou y Ramón-Muñoz, 2011).

\subsection{La modernización como excusa: los riegos tradicionales del río Muga y del Bajo Ter}

La llanura del Ampurdán, extendida genéricamente desde el sur del río Ter hasta el norte del río Muga, caracteriza un territorio que históricamente ha permitido el desarrollo de cultivos tanto en régimen de secano como de regadío (ver figura 1). No obstante, los primeros canales de una cierta entidad, que se remontan al final de la edad media, se construirán, no tanto para la dotación de riego, como para proporcionar la fuerza hidráulica a los molinos, preferentemente harineros, que se instalarán en sus inmediaciones. De ahí se explica que tanto en la cuenca de la Muga, como del Fluviá, como del Ter, las denominaciones de los canales históricos concuerden con las de los respectivos molinos para los que fueron proyectados (recs o sèquies del molí). Son los casos, por ejemplo, del rec dels molins de Cabanes y Peralada (río Muga), del rec dels molins de Valveralla y L’Armentera (río Fluviá) o de la sèquía dels molins de Gualta y Pals (río Ter). Estos canales han configurado, por lo menos durante medio milenio, las arterias principales del regadío tradicional para la huerta, el arroz, la alfalfa, el maíz o los frutales, entre otros. Tanto es así que los recs o sequies se han integrado como unos elementos territoriales y del paisaje que van más allá de su función estrictamente productiva. Se trata de unas construcciones sin revestimiento que, pese a su carácter antrópico, también juegan una función desde la perspectiva ambiental -refugio para la flora y fauna propias de estos ambientes húmedos, conectores biológicos, regulación de acuíferos, etc. Además, otorgan una cierta personalidad a los núcleos históricos de las poblaciones que flanquean -como Castelló d'Empúries o Verges- y sirven como trazado para itinerarios pedestres y en bicicleta. Así que, la dimensión socio-cultural de estos canales resulta innegable aunque, con demasiada frecuencia, su abandono y falta de mantenimiento repercuta en la degradación de diversos puntos de su recorrido. El deseo de consolidar la superficie de riego y sus dotaciones conducirá a que, desde el momento en que se disponga de los medios tecnológicos y económicos suficientes, se promuevan grandes proyectos de irrigación con una red de canales sistematizada y jerarquizada asociada a la ejecución de grandes embalses en los respectivos cursos fluviales.

Desde la segunda mitad del siglo XIX se enumeran diversas propuestas que, de manera recurrente, fijan su horizonte en una llanura del Ampurdán irrigada casi en su totalidad, gracias a extensas redes de riego interconectadas. Un riego que ha de suponer bondades económicas y sociales de todo tipo, casi regeneracionistas, para el sector agrario, en particular, y para el conjunto de la zona, en general. Es la visión maximalista de las 50.000 hectáreas de riego que también subyace en el momento de ofrecer medidas compensatorias al trasvase del Ter hacia Barcelona y sus proximidades, a finales de la década de 1950. De hecho, será la derivación de los caudales del Ter a Barcelona, pero también los episodios de inundaciones y los planes desarrollistas de la década siguiente los que van a permitir materializar, parcialmente, la ejecución de una nueva red de regadío modernizada, asociada a grandes embalses y a sus respectivos azudes de derivación. La progresión que tendrán estas obras, a partir de entonces, va a conducir a las tres cuencas de la llanura del Ampurdán a una situación diferente desde la perspectiva del riego y de su modernización (Pavón, 2008).

\subsubsection{Los regadíos de la cuenca del río Muga}

En el caso de la cuenca de la Muga, se ejecutará el embalse de cabecera (Boadella), el azud de derivación (Pont de Molins) y un porcentaje significativo de la superficie de riego (unas 6.000 de las 10.000 hectáreas previstas). Esto va a comportar el abandono o casi abandono de los antiguos canales de riego y su substitución por una nueva red mucho más extensa, con estructuras aéreas y prefabricadas. El crecimiento urbano de Figueres, la expansión de los asentamientos turísticos del litoral (Empuriabrava, Sta. Margarida,...) o los requisitos ambientales del parque natural dels Aiguamolls de l'Empordà (PNAE) serán variables que van a condicionar la progresión de estos regadíos hasta el presente. Una muestra definitiva de la confrontación por lo que se refiere a los aprovechamientos de los recursos hídricos se vivió a partir de 1984, con la explotación del acuífero del curso bajo del río Muga, con duros enfrentamientos entre regantes y el sector turístico, en la denominada "guerra de los pozos" y que desencadenó, a su vez, 
importantes conflictos entre los municipios implicados. Así, por ejemplo, mientras que un municipio como Castelló d'Empúries esgrimía su derecho a retener el agua en su territorio, otros más alejados, como Roses y Cadaqués hablaban del deber de aquel municipio de compartir el agua con sus vecinos (Ventura, 2004). La extracción creciente de los pozos para atender las demandas turísticas estacionales justo en el momento álgido de los riegos agrícolas provocó su salinización y la correspondiente confrontación por dirimir qué aprovechamientos debían de prevalecer sobre los demás. La solución adoptada fue la construcción de una planta potabilizadora en el acceso de la urbanización Empuriabrava que tratase el caudal del centenario Rec del Molí, que sería derivado, a su vez, des del río Muga. Aunque la instalación entró en funcionamiento en 1987, poco después, en 1989, la captación se pasó a realizar directamente desde el canal principal del margen izquierdo del río Muga, con la construcción de un depósito acumulador de $20.000 \mathrm{~m}^{3}$ de capacidad que, por gravedad, conecta con la conducción del Rec del Molí. La gran urbanización de Empuriabrava, así como Roses, Cadaqués, Llançà y una mancomunidad de municipios de menor rango demográfico se irán conectando, progresivamente, a estas instalaciones. De esta forma, junto con Figueres y los núcleos próximos, el conjunto de municipios anteriores pasaba a ser el principal abastecimiento urbano dependiente del embalse y configuraba el sistema "Costa Brava Norte".

Figura 1. Ámbitos de localización del regadío ampurdanés

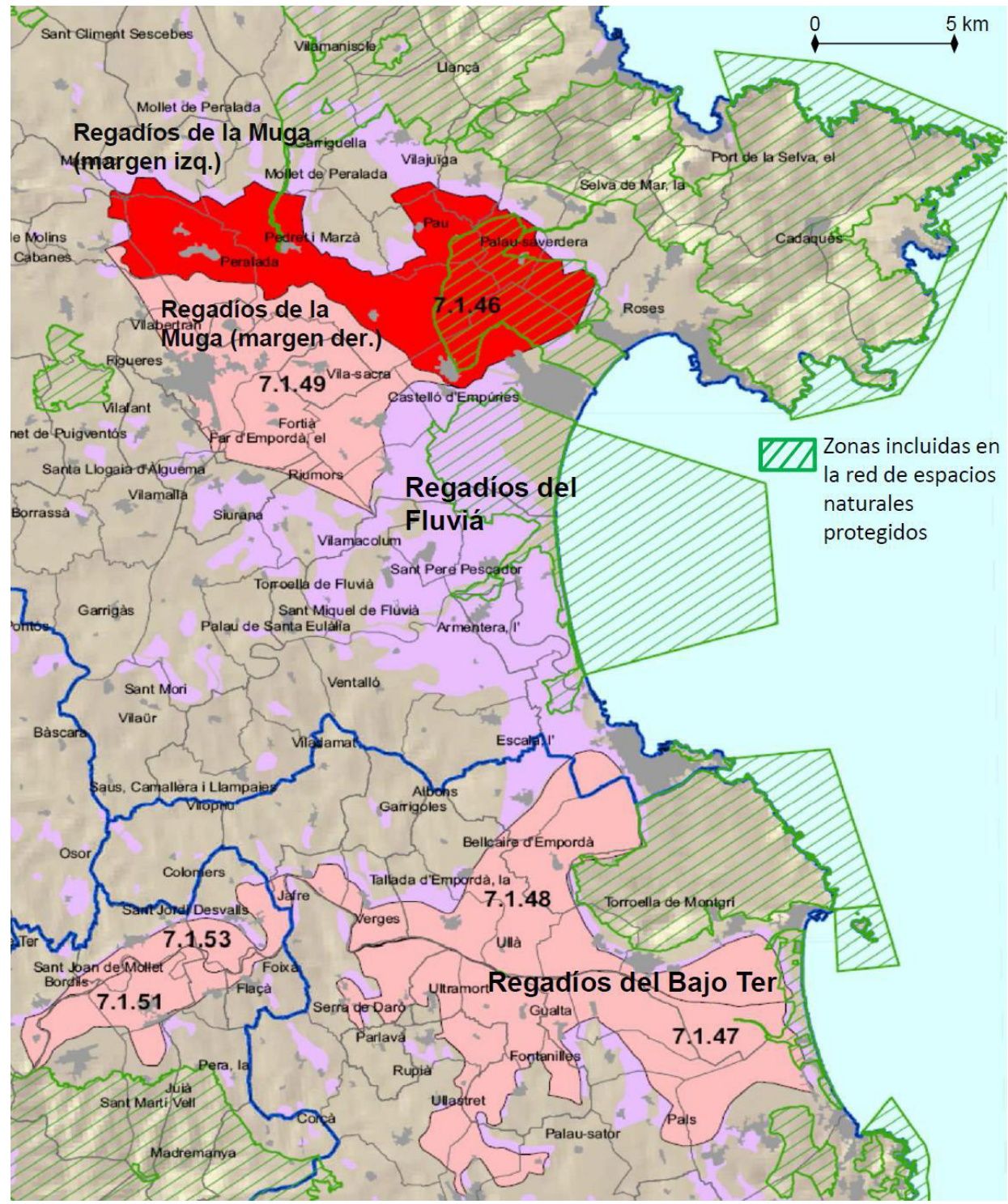

Fuente: adaptación a partir de IGREMAP, S.L.P. (2010): Pla de Regadius de Catalunya 2008-20 
Complementariamente a los usos turísticos y urbanos en sus diversas facetas, a partir de la década de 1980 tomó relevancia la reserva de una parte de las aportaciones del río Muga para usos ambientales y que tenían por objetivo conservar, en la medida de lo posible, los ecosistemas acuáticos. Un objetivo crucial será evitar el descenso de la línea piezométrica y la consiguiente salinización, por invasión del agua marina, del freático asociado al curso bajo del río. La creación del Parque Natural dels Aiguamolls de l'Empordà (PNAE), en 1983, precisará de una disponibilidad de agua que se ajuste a sus objetivos de protección y regeneración de las áreas lacustres. Así, se ha convertido en un nuevo "cliente" de las aguas del embalse. La alimentación hídrica del PNAE tiene diversos orígenes: aguas superficiales que llegan directamente de los ríos Fluviá y Muga, aguas procedentes de los acuíferos de ambos ríos, aguas tratadas en la estación depuradora de Empuriabrava y, indirectamente, el propio embalse de Boadella, mediante los canales de irrigación que acababan dentro del Parque. Según la Agencia Catalana del Agua (ACA), administración hidráulica competente en las cuencas interiores de la comunidad autónoma, el caudal ecológico desembalsado en los últimos años por la presa, si sitúa alrededor de los 150 1/s i se entiende que, en todo momento, deberían circular, por lo menos, 135 l/s. Si se da por buena la cifra mínima de 135 l/s, se obtienen $4,26 \mathrm{hm}^{3} /$ año reservados para el caudal ecológico. El Plan sectorial de caudales en las cuencas internas de Cataluña ${ }^{5}$ recoge que el caudal ecológico se debiera modular, mensualmente, entre 270 y 410 1/s. Son, por tanto, unas cifras que podrían llegar a doblar los niveles habituales.

Según los datos de la ACA (ACA, 2009), para el período 1940-2008 las aportaciones medias anuales de la Muga han sido de unos $147 \mathrm{hm}^{3}$, de los que aproximadamente un $40 \%$ son susceptibles de ser regulados por el embalse de Boadella. El régimen de estas aportaciones es extremadamente irregular, de manera que hay muchos años en que los recursos se sitúan por debajo de la mitad de la media, en épocas que se pueden considerar de sequías graves. Durante estos episodios, la capacidad de regulación del embalse respecto las demandas que dependen de él es muy escasa.

Las demandas vinculadas directamente a Boadella son, según la ACA, de poco más de $40 \mathrm{hm}^{3}$, aproximadamente la mitad de la demanda total del sistema Muga. El resto de aprovechamientos son mayoritariamente de aguas subterráneas de los diferentes acuíferos del ámbito, entre los que destacan los aluviales de la zona de Peralada, donde captan diferentes redes de abastecimiento, y el fluvio-deltaico compartido con el Fluviá, donde las extracciones, más dispersas y, en algunos casos afectadas por la salinización de la zona más litoral, son básicamente de regadíos. Se ha de aclarar que, además de las 5.000 ha que son regadas de manera efectiva a través de los caudales subministrados desde el embalse, hay alrededor de 6.300 ha dispersas en el resto de la cuenca, emplazadas, mayoritariamente, al sur del río Manol y que se alimentan fundamentalmente de pozos. Aquí se incluyen los regadíos de los sectores VI y VII, en el margen derecho de la Muga, que en un principio se debían atender con aguas del embalse.

El peso creciente de los abastecimientos urbanos ha contribuido que, por parte de la administración hidráulica, se fijen unos umbrales para su garantía y que fluctúen según el nivel de agua disponible en el embalse. Tanto los usos agrícolas como los ambientales padecerían restricciones al abastecimiento, en diversa medida, con el fin de evitar afectaciones severas que pudiesen incidir sobre la demanda urbana. Así, según los datos de la ACA, la garantía actual de los abastecimientos de Figueres y del Consorcio de la Costa Brava norte es prácticamente del $100 \%$ al establecer una curva de reserva en Boadella que se apoya sobre las demandas de riego. Esta curva de reserva hace que, aproximadamente, cuando el volumen almacenado en el embalse baja del $40 \%$ de su capacidad máxima y se pasa a un escenario de gestión de alerta según el Plan de gestión de sequías, se fijen restricciones sobre las demandas no prioritarias -regadíos- y se reduzcan los caudales ambientales para garantizar el subministro de los abastecimientos. En situación de alerta, estas restricciones son, aproximadamente, de un 25\% respecto la demanda en condiciones ordinarias o de normalidad, en el caso de los regadíos, y de un 36\% para los caudales de mantenimiento del Plan sectorial, asegurando siempre un hábitat potencial útil superior a un mínimo del 30\%. En caso de empeoramiento de esta situación, en el siguiente escenario de excepcionalidad, con reservas aproximadas por debajo del $20 \%$, las restricciones se incrementarían a un $80 \%$ de las demandas en normalidad, tanto en el caso de los regadíos como en el de los caudales ambientales (ACA, 2009). Estas

5 El Plan se presenta como el instrumento para conseguir un buen estado ecológico de las masas de agua, en cumplimiento de los objetivos establecidos por la Directiva Marco del Agua (2000/60/CE). 
restricciones y su repercusión más directa en el sector agrario han generado fricciones con los regantes, que han de modular la superficie de riego de cada campaña así como el tipo de cultivo que se planta.

La situación que se vive en la cuenca del río Muga con la dependencia de una única gran infraestructura hidráulica y de los acuíferos obliga a la adopción de medidas que pasen por el ahorro y el aumento de la eficiencia en los consumos hídricos. El Plan de Gestión del distrito de cuenca fluvial de Cataluña, elaborado por la ACA en el año 2009, es la herramienta que ha de determinar las acciones y las medidas necesarias para desarrollar los objetivos de la planificación hidrológica del distrito de cuenca fluvial de Cataluña. Su ámbito territorial está constituido por las cuencas hidrográficas internas catalanas y por las aguas subterráneas y costeras asociadas ${ }^{6}$. Para el caso de la cuenca de la Muga, el Plan de Gestión preveía, hasta el año 2015, un crecimiento de la demanda para usos urbanos del orden de $1 \mathrm{hm}^{3}$ i un cierto potencial de ahorro para los regadíos, que se podría situar entre los 4 y los $6 \mathrm{hm}^{3}$ respecto la situación actual. Para la irrigación, la capacidad de ahorro provendría de la modernización de su tecnología y de la reutilización de los caudales regenerados de las depuradoras (fundamentalmente de Figueres y Roses). Esta última actuación se debería plantear de manera conjunta con actuaciones de recarga y explotación de las aguas subterráneas. El Pla admite un posible recrecimiento de Boadella de unos $12 \mathrm{hm}^{3}$, pese a reconocer que solo ofrecería beneficios algunos años secos, después de los más húmedos. Contrariamente no considera factible la expansión del actual dominio regable del canal principal de la Derecha (sectores VI y VII pendientes) por su baja garantía y por la presión que ocasionaría sobre el resto del sistema. En cuanto a los caudales ecológicos, se valora el escenario en que su caudal oscilase, en función de la época del año, entre los 270 y los 410 l/s; por tanto, al menos el doble que el habitual. Cabe señalar, no obstante, que la redacción del citado plan todavía se hace a reflujo de la etapa de expansión económica producida en los años anteriores. Por otra parte, la crisis posterior ha supuesto una profunda revisión de las posibilidades de financiación de las actuaciones previstas en el plan, aspectos que acentúan la incertidumbre en el devenir de la cuenca.

En la última década, ya con anterioridad a la aprobación del plan, se han ejecutado medidas como la construcción de balsas reguladoras para las escorrentías de los canales o la sustitución progresiva de la red aérea construida a partir de la década de 1960, por otra de tipo subterráneo. Hasta el momento ha quedado sin efecto el posible suministro mediante efluentes de las depuradoras urbanas.

\subsubsection{Los regadíos del Bajo Ter}

En el caso del río Ter, aunque se construyó el complejo de embalses (Sau-Susqueda-El Pasteral) así como el azud de compuertas en Colomers, ha quedado sin ejecutar la red de canales tanto del margen izquierdo como del derecho. A diferencia de la cuenca del río Muga, son los canales históricos de Sentmenat (margen izquierdo) y de Gualta-Pals (margen derecho) los que continúan abasteciendo a los regantes, con unas 6.500 ha. en total. Quizás sean estos dos canales los que mejor se ajustan a la idea de multifuncionalidad que comentábamos anteriormente. En el caso de la acequia de Gualta-Pals, a partir del año 2004, se van a plantear diversos proyectos de modernización con la pretensión de reducir los caudales aportados a la irrigación, así como las labores de mantenimiento de la acequia. La solución adoptada, a partir de 2007-08, pasará por el entubamiento de su trazado con el objetivo de reducir, según los cálculos, entre 7-10 hm³, al suprimir las filtraciones desde la acequia (ver figura 2). No obstante, este planteamiento ha contado con la oposición de organizaciones ambientalistas e incluso de un sector de los regantes por el elevado coste económico -28 millones de euros-, así como por la pérdida de funcionalidad y por los efectos ecológicos que tendría sobre la acequia, en su lámina de agua circulante y en el propio freático. Se cuestiona que el supuesto ahorro previsto, posteriormente pueda dirigirse al incremento de nuevos regadíos sin las garantías suficientes o que, incluso, pueda ser utilizado no para que revierta en el territorio, sino para aumentar la garantía en las aportaciones desde los embalses del Ter hacia Barcelona y sus alrededores. Por otro lado no queda claro el supuesto ahorro hídrico si, como recoge la declaración de impacto ambiental del proyecto (años 2007 y 2008), deben respetarse unos caudales ambientales en la

6 El Plan fue aprobado el 16 de setiembre de 2010 por el Consejo de Administración de la ACA por 19 votos a favor y 7 en contra. 
acequia (200 1/s). Todo ello sin contar con la desestabilización como regulador en el freático que, hasta el momento, ha ejercido la acequia histórica (Ribas et al., 2011).

Figura 2. Construcción de la nueva conducción de agua para los riegos del bajo Ter, a la derecha de la acequia histórica de Gualta-Pals, en el punto de derivación de sus caudales respecto al propio río Ter (arbolado en la izquierda de la imagen). Mientras las obras se ejecutan, unos tubos provisionales permiten que el agua continúe discurriendo a través de la acequia histórica a cielo abierto.

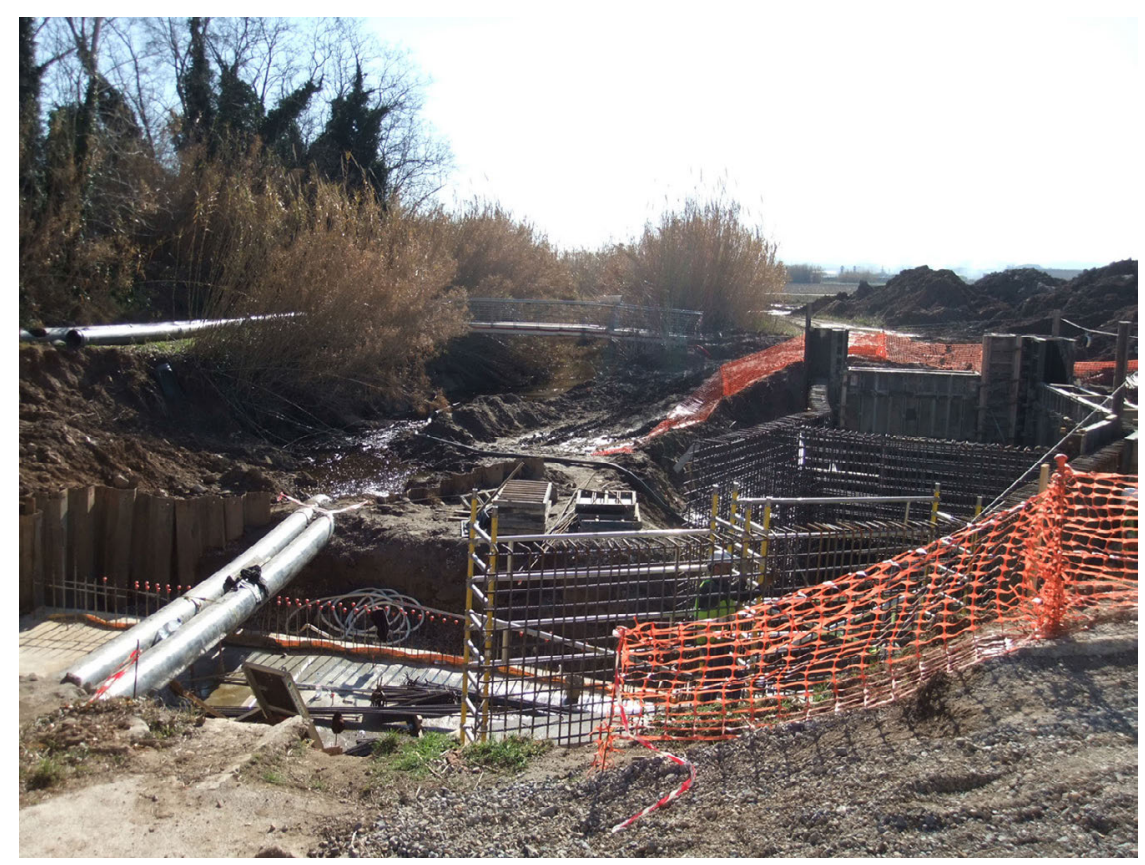

Autor: D. Pavón (24-I-2012)

En el caso del río Fluviá, la ejecución de grandes obras hidráulicas ha quedado sin efecto. No se ha materializado ni el embalse regulador (Crespiá), ni el azud principal, ni la red de canales. Pese a ello, en la actualidad el regadío ocupa una extensión significativa y se efectúa mediante bombeos del freático.

\subsection{El canal Segarra-Garrigues: de la reivindicación al desencanto y de éste al conflicto}

Si existe un canal de regadío capaz de recibir tantos calificativos como sumar dificultades y demoras en su puesta en marcha, seguramente ese sea el canal Segarra-Garrigues. Desde su carácter faraónico al reflejo de la discordia y el conflicto de intereses, el canal Segarra-Garrigues da respuesta a una reivindicación histórica del campo leridano fruto de los paralelismos con los regadíos existentes del Urgell impulsados ya en el siglo XIX. Un canal de largo recorrido, contemplado por la Generalitat republicana en la década de 1930, fortalecido con su inclusión en el Plan de aprovechamiento total del río Segre del 1959 , debatido internamente a lo largo de los años setenta y ochenta ${ }^{7}$ y reconocido como infraestructura de interés general en $1994^{8}$. Un año más tarde se redactará la memoria descriptiva de la zona regable del canal. En 2002 se publicará el Proyecto de regadío y de concentración parcelaria y se redactará la Declaración de Impacto Ambiental no sin dificultades, pues parte del recorrido del canal se verá afectado por la inclusión de Zonas de Especial Protección para las Aves (ZEPA) en buena parte de su recorrido. Dicho contratiempo no impedirá el inicio de las obras del canal que en la actualidad están en su fase de ejecución definitiva, con parte del canal ya en funcionamiento9. Un canal que recogerá los imaginarios

7 Con reuniones informativas en diferentes localidades leridanas donde representantes institucionales, sindicales y payeses reclamarán un aprovechamiento integral del río Segre para regar cuantas más hectáreas mejor.

8 Será este un momento que impulsará definitivamente el proyecto y con ello el mismo se reconocerá en los Planes posteriores: el Plan Hidrológico de la Cuenca del Ebro, el Plan Nacional de Regadíos y el Pla de Regadius de Catalunya.

9 Durante la Fira Agraria de Lleida en septiembre de 2013, el ministro de Agricultura aproximará la complementación de los dos últimos tramos del canal para 2014 mientras que la finalización del embalse del Albagés se espera para 2016. 
colectivos de una sociedad y de un determinado modelo de desarrollo y que tenderá a calificarse como un conflicto de intereses bifocal entre los promotores y los defensores del regadío y aquellos que priorizarán la preservación de las estepas de Ponent y la dinámica ambiental ${ }^{10}$.

El sistema Segarra-Garrigues abarca cerca de cien mil hectáreas de superficie bruta de un total de 73 municipios de las comarcas de la Noguera, la Segarra, l'Urgell, el Pla d'Urgell, les Garrigues y el Segrià ${ }^{11}$. Según el proyecto de regadío, estas comarcas recibirán el agua mediante el canal desde el embalse de Rialb hasta el embalse de nueva construcción del Albagés y de dos captaciones al río Segre emplazadas en los municipios de Albatàrrec y de Aitona con el fin de elevar el agua del Baix Segre (ver tabla 1). Unos bombeos que requerirán una potencia eléctrica que la empresa Endesa gestionará mediante la construcción de subestaciones eléctricas y nuevas líneas de transporte y distribución, además del acondicionamiento de las infraestructuras eléctricas existentes. En este sentido, la factura energética del canal será otro de los aspectos que dificultará la puesta en regadío efectiva de un número de hectáreas mayor.

Tabla 1. Principales cifras del sistema Segarra-Garrigues

\begin{tabular}{|l|l|}
\hline \multicolumn{1}{|c|}{ Características } & \multicolumn{1}{|c|}{ Afectación } \\
\hline Río & Segre \\
\hline Superficie bruta del sistema & 105.000 hectáreas \\
\hline Superficie de regadío afectada por el canal & 70.150 hectáreas \\
\hline Embalses implicados & Rialb y Albagés \\
\hline Captaciones de agua & Albatàrrec y Aitona \\
\hline Comarcas & 6 \\
\hline Municipios & 73 \\
\hline Consumo de boca & 72.000 habitantes \\
\hline Regantes potenciales & 16.000 \\
\hline Sectores de riego & 15 \\
\hline Capacidad de transporte & $15-35 \mathrm{~m}^{3} / \mathrm{s}$ \\
\hline Caminos rurales de nueva construcción & $1.500 \mathrm{~km}$ \\
\hline Concentración parcelaria & 48.000 hectáreas \\
\hline Mejora de infraestructuras eléctricas & $180.000 \mathrm{~kW}$ nuevos \\
\hline Rehabilitación del patrimonio histórico (1\%) & $10 \mathrm{M} \mathrm{EUR}$ \\
\hline Red Natura 2000 & 41.500 hectáreas \\
\hline Dotación anual para el canal & $342 \mathrm{hm}{ }^{3}$ \\
\hline Dotación de transformación $\left(6.500 \mathrm{~m}^{3} / \mathrm{ha} / \mathrm{año}\right)$ & 47.110 hectáreas (margen derecho) \\
\hline Dotación de soporte (3.500-1.500 m $\left.{ }^{3} / \mathrm{ha} / \mathrm{año}\right)$ & 23.040 hectáreas (margen izquierdo) \\
\hline Tuberías & $3.500 \mathrm{~km}$ \\
\hline Balsas de regulación & 43 \\
\hline Estaciones de bombeo & 17 \\
\hline Presupuesto del canal principal y del embalse de Albagés & $444 \mathrm{M}$ EUR \\
\hline Presupuesto de la red secundaria & $1.069 \mathrm{M}$ EUR \\
\hline
\end{tabular}

Fuente: Elaboración propia

10 Es interesante la distinción que hace Muñiz (2010) entre el convencimiento del canal Segarra-Garrigues desde la razón social y económica y el énfasis en la legitimidad legal que supone la contribución catalana a la red europea Natura 2000. Así mismo, la autora concluye (2010: 24): "suelen ser las ideas estereotipadas que tienen unos y otros los que marquen la confrontación. El tópico del payés que no se preocupa del entorno natural y a quién solo le interesa producir y quiere "agua y más agua" y que choca con el tópico del ecologista que es proteccionista por encima de todo y a quién sólo le agrada la natura "deshumanizada" y quiere "aves y sólo aves"”.

11 Según lo expuesto en la memoria socioeconómica elaborada por el Departamento de Agricultura, Alimentació i Acció Rural (DAR) en 2010 para la construcción del canal Segarra-Garrigues, la población activa agraria representa, de media, el 16,5\% del total poblacional -cuando a nivel de país representa menos del 3\%. En referencia al PIB, si bien en Cataluña el sector agrario representa cerca del $2 \%$ del PIB total, en las seis comarcas del canal el porcentaje sectorial difiere entre el 15 y el 20\%. En cuanto a la caracterización del sector, del total de 8.000 explotaciones contabilizadas, dominan las explotaciones en propiedad (80\%) y de menos de 4 hectáreas. 
Será un proyecto pensado para dinamizar el sector agroalimentario de la zona y promover su desarrollo económico, si bien la multifuncionalidad obligada a la que deberá hacer frente seguirá ocupando el debate en torno a su desarrollo y gestión. Así, la coincidencia en tiempo y espacio entre la expectativa de riego y la conservación esteparia será el primer factor de conflicto al cual le seguirán la propia viabilidad económica del canal así como la implicación de la sociedad civil mediante el protagonismo de los firmantes del Manifest de Vallbona ${ }^{12}$ y su evolución hacia el Compromís per Lleida y el colectivo Aigua per Unir. Con dicho manifiesto el colectivo recogerá cuatro aspectos clave en la consecución del canal de modo satisfactorio: a) el conjunto de actuaciones relacionadas con el canal no se deben considerar una mera transformación de regadío sino como una herramienta imprescindible para la política de ordenación territorial del secano leridano; b) es necesario y urgente promover una nueva estructura agraria que facilite el relevo generacional desde la promoción de la actividad agraria de regadío como un mecanismo de gestión y conservación del territorio; c) cabe incorporar al proyecto los postulados de la Nueva Cultura del Agua para priorizar la eficiencia hídrica de la actividad agrícola, valorar el bagaje patrimonial y cultural de su práctica y vincular el carácter productivo al desarrollo rural del territorio; y d) el coste de la actuación en regadío no puede ser asimilado únicamente por el agricultor pues el conjunto de la sociedad es beneficiaria de los servicios ambientales de su actividad.

\section{EL PAPEL DE LAS DEMANDAS SOCIALES EN LA GESTIÓN DEL REGADÍO: MOTOR DE CAMBIO Y DE CONFLICTO}

La percepción social sobre el papel del regadío difiere en función de la perspectiva desde la cual se analizan los pros y contras de su modernización y/o promoción. Sus efectos sobre la dinámica territorial, el medio ambiente o el paisaje así como su significancia como usuario máximo de recursos naturales como son el agua y el suelo o su capacidad estratégica en promover la seguridad alimentaria, generan controversia (ver figura 3) (Oca, 2009). Si bien la modernización de regadíos históricos suele ser vista como una práctica necesaria en pro de una mayor eficiencia hídrica que contribuya a la mejora cualitativa y cuantitativa del caudal ecológico de los ríos -como en los riegos del Bajo Ter y de la Muga-, las actuaciones que conllevan nuevos regadíos -como el canal Segarra-Garrigues- suelen requerir un grado de aceptación y de consenso social mayor. Éstos serán difícilmente asumibles sin abrir el debate sobre el regadío al conjunto de sectores económicos y demás usos consuntivos del agua así como a las consideraciones de la sociedad civil acerca del modelo de desarrollo del territorio ${ }^{13}$ (Aldomà, 2012).

No hay duda de que la percepción del regadío ha cambiado, tanto desde el propio sector como, sobre todo, desde la ciudadanía. El regadío ya no es, per se, un factor de desarrollo socioeconómico. De hecho, son notables los ejemplos de proyectos de regadío que no han cumplido las expectativas generadas. La sensibilización ciudadana e institucional crece en torno a la multifuncionalidad del regadío, de tal forma que los valores patrimoniales, lúdicos, educativos y ambientales vinculados al regadío también son asociados a la estrategia a seguir para dinamizar el conjunto del territorio (Brunstad et al., 2005). Con ello, la gestión del regadío se enfrenta a retos complejos y dinámicos. Como usuario dominante del recurso agua debe hacer frente a variables como el cambio climático, la seguridad y soberanía alimentarias, los condicionantes ambientales, las demandas sociales de participación y la compatibilidad de usos en competencia.

12 Firmado en el mes de mayo de 2004 en el municipio leridano de Vallbona de les Monges por parte de 150 representantes de los sectores agrario, cooperativo, sindical, profesional, intelectual e institucional de Lleida con el objetivo de fomentar el debate de alternativas sobre el futuro del canal. Temas como el replanteamiento del proyecto teniendo en cuenta la ordenación del territorio, la necesidad de una nueva financiación, las repercusiones en la reestructuración del sector agrario, la inclusión de los postulados de la Nueva Cultura del Agua o la constitución de un fondo de tierras para facilitar el acceso de los jóvenes a la agricultura serán parte de las temáticas a tratar. Unas cuestiones que quedarán resumidas en la formulación de los siguientes interrogantes sobre el futuro del canal: ¿Con qué agua?, ¿Para fortalecer qué tipo de modelo agrario?, ¿Para qué territorio? o ¿Para qué tipo de sociedad?

13 Un ejemplo de oportunidad ante la diversidad de usos que soporta el canal será la propuesta de creación de un Consorcio del Albagés con el objetivo de aprovechar turísticamente el embalse del Albagés y su entorno natural. Con ello, el canal puede ser el elemento articulador de una bioregión donde se imponga la cooperación entre sectores productivos, servicios lúdicos y educativos y buenas prácticas en materia de sostenibilidad ambiental. Así, el territorio-paisaje del canal podría considerarse una vía verde que resiguiera la geografía de las concentraciones parcelarias, de las áreas de protección ambiental, de los sistemas hídricos o de los itinerarios patrimoniales. 
Figura 3. La percepción social del regadío: efectos, retos y variables

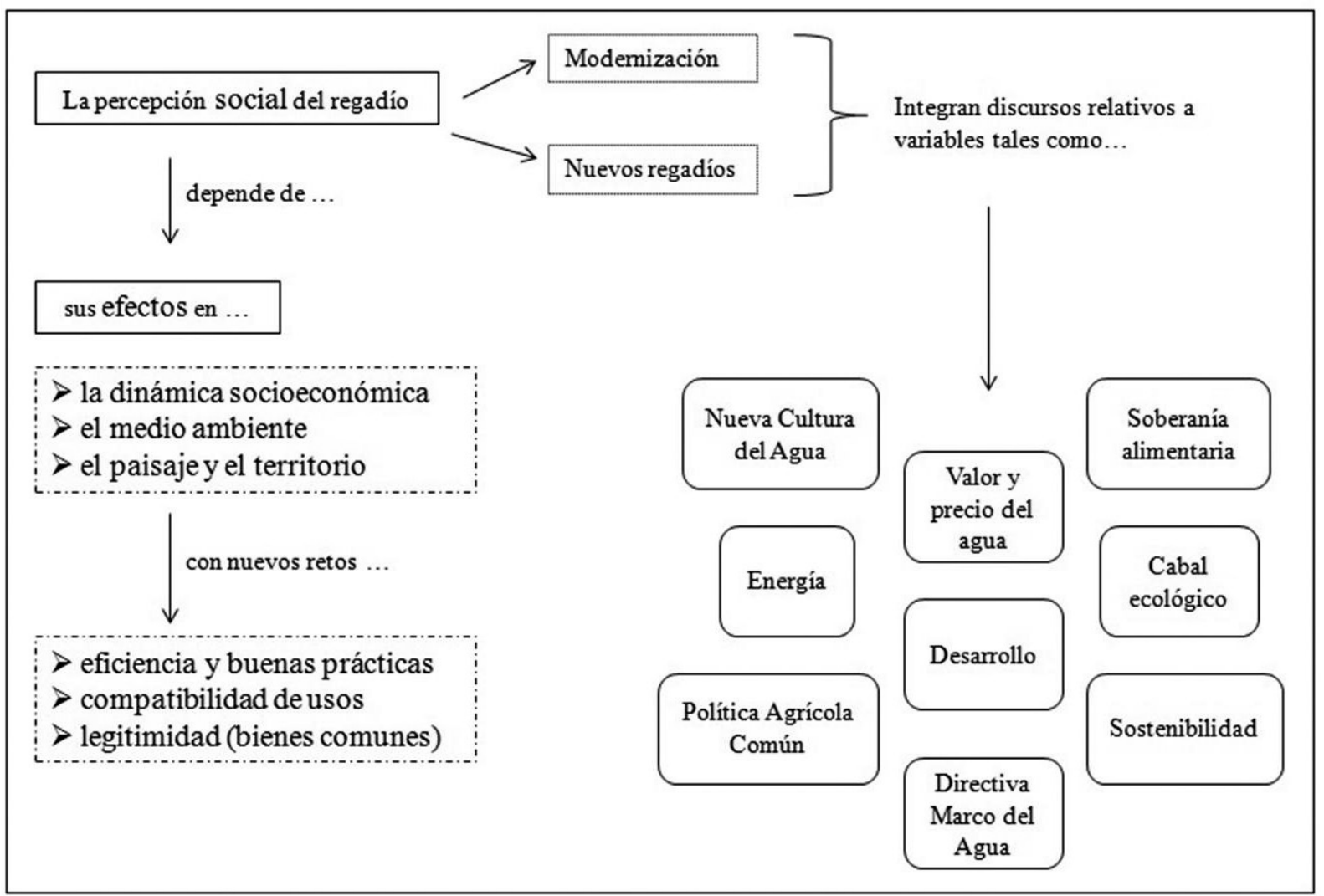

Fuente: Elaboración propia

\section{4. ¿EL FUTURO DEL REGADÍO? GESTIONAR LA COMPLEJIDAD DESDE LA GOBERNANZA}

La complejidad que envuelve la gestión del territorio conlleva aceptar el dinamismo de ciertas variables: la disponibilidad de recursos naturales como el agua y el suelo, la competencia y/o conflicto entre usuarios y regiones, la preocupación por los aspectos ecológicos o la legitimación social de todo proyecto o actividad de afectación multisectorial, como es el regadío. Una práctica que, con el paso del tiempo, ha convertido los objetivos iniciales en auténticos retos cada vez más complejos -como la seguridad alimentaria o la gestión sostenible de los recursos hídricos (Groenfeldt, 2006). Cierto es que la gobernanza en la gestión del regadío confronta intereses y prioridades específicas dadas las características intrínsecas de los diferentes actores sociales implicados (Ricart et al., 2011). Tradicionalmente, ha sido el Estado quien ha capitaneado la gestión del riego, erigiéndose como el principal planificador e interventor sobre un regadío concebido como principal factor de desarrollo. En paralelo, la implicación gradual del sector privado como ejecutor de dichas políticas y proyectos ha permitido hacer frente el desembolso económico que toda infraestructura de regadío -algunas de carácter faraónico- conlleva y, al mismo tiempo, dar lugar a un cierto grado de privatización del uso y práctica del agua. Cerrando este triángulo relacional se sitúa un tercer actor representativo de forma directa del sector productivo agrario, la comunidad rural, vinculada, principalmente, a sindicatos y comunidades de regantes.

Sin embargo, con el paso del tiempo y basándose en los cambios experimentados en el enfoque de la gestión de los recursos hídricos, el regadío ha visto como en los últimos años nuevos actores reclaman cierto grado de protagonismo. Destaca principalmente un actor, la sociedad civil organizada, que a partir de criterios básicamente ambientales -aunque no exclusivamente-, cuestiona la legitimidad del modelo de gestión tradicional del regadío por una falta de gobernanza en sus postulados. Se trata de una reivindicación que reclama muchas veces una mayor implicación de la sociedad en temas que rebasan un interés meramente sectorial, como sería el regadío, para aunar en sus cada vez más reconocidos 
valores ambientales, paisajísticos y culturales. Así, empiezan a ser habituales los ejemplos de cómo parte de la sociedad civil se moviliza y se postula como integradora de las nuevas funciones y usos vinculados al regadío y su previsible afectación en la dinámica territorial, integrándose en el debate sobre su evolución futura. Sólo por citar algunos ejemplos, vale la pena destacar, a nivel nacional, las principales organizaciones ecologistas que integran el Consejo Asesor de Medio Ambiente del Ministerio de Agricultura, Alimentación y Medio Ambiente -WWF, Greenpeace, Ecologistas en Acción, Amigos de la Tierra, SEO/Birdlife-; las plataformas ciudadanas dedicadas a la defensa de los ríos que abastecen buena parte de las principales zonas de regadío de la Península Ibérica -Plataforma del Guadalquivir, Red Tajo, Ojos del Guadiana Vivos, Plataforma en Defensa del Ebro, Plataforma del Ter- o las que aglutinan a un conjunto de sectores diversos de la sociedad -Compromís per Lleida o Aigua és Vida.

\subsection{El papel de la política europea: síntomas de cambio a favor de la gestión integrada}

A nivel político y en el marco europeo, la cuestión que persigue respuesta es: ¿podemos gestionar el regadío como suma de producción alimentaria, dinámica ambiental y participación social? Los promotores de la última reforma de la Política Agrícola Común (2014-2020) así lo consideran y para ello proponen un instrumento específico: el greening o ecologización. Un concepto que, a grandes rasgos, supone un paso más del iniciado en la década de los noventa con las medidas agroambientales de la reforma MacSharry pero que no deja de reivindicar el vínculo indisociable entre la satisfacción de las necesidades alimenticias, su repercusión en los requerimientos ambientales y la legitimación social de su práctica (Ricart y Roca, 2013). Pero no es la única política de ámbito europeo que enfatiza en la necesidad de gestionar el medio ambiente como parte integrada de las prácticas sectoriales. Los Programas de Acción para el Medio Ambiente se han ido configurando, desde el primero de ellos en 1973, como un instrumento de referencia en la integración de los retos que superan el enfoque sectorial como el regadío. Precisamente el último programa (2013-2020) pone el énfasis en los recursos naturales -y en especial, el agua y el suelo- y su gobernanza implícita como única opción ante la creciente conflictividad de intereses que conlleva la disponibilidad o carencia de ambos. Por su parte, la Directiva Marco del Agua también condiciona el modelo de desarrollo del regadío en priorizar el buen estado ecológico de los ríos mediante la gestión integrada y la participación ciudadana ${ }^{14}$. Así pues, dichas políticas suman variables para reconocer la naturaleza holística del ciclo del agua desde la eficacia de las instituciones competentes para sumar competitividad, participación social y legitimidad a la toma de decisiones en materia de regadío.

\subsection{La gobernanza de los recursos hídricos: primer paso para legitimar el regadío}

Ante una situación de competencia y complejidad casi a partes iguales, la promoción de un concepto polisémico como es la gobernanza y su vínculo con la gestión de los recursos hídricos se ha situado en el centro del debate (Pahl-Wostl, 2010). Para autores como Dore et al. (2012) dicho binomio se concibe desde una doble vertiente: la social, como un proceso de diálogo, de negociación y de toma de decisiones, y la instrumental, como un medio para alcanzar unos objetivos predeterminados. Un compromiso entre la coordinación de las acciones individuales y las formas de gestión colectiva, es decir, una nueva forma de organizar el poder y de gobernar la sociedad (Tropp, 2007). Cierto es que no estamos acostumbrados a gestionar desde la adaptación y/o proposición sino desde la reacción y que, difícilmente, se relaciona la participación y la cooperación con la mejora de la toma de decisiones (Allan y Wouters, 2004). La concepción positiva de la movilización ciudadana como ejemplo de gestión bottom-up, la voluntad de establecer un pacto que facilite el acuerdo entre posturas distantes o la capacidad política de definir un debate público capaz de analizar un proyecto desde diferentes puntos de vista sirven de ejemplos de cómo la gobernanza puede beneficiar la gestión del regadío. En pocas palabras: se trata de gestionar la complejidad aceptando, primero, la evolución de condicionantes como la disponibilidad de

14 Cabe señalar aquí el doble papel de la política española de regadíos en relación al primero de ellos, el buen estado ecológico. Así, si por un lado los proyectos de modernización de regadíos tradicionales, en pro de la eficiencia en el uso agrícola del agua, benefician la dinámica fluvial de los ríos prestadores del agua en limitar el requerimiento de riego, por el otro los nuevos proyectos de regadío suponen muevas tomas de agua, repercutiendo en la dinámica ecológica del mismo al disminuir su cabal. 
recursos naturales; segundo, el mantenimiento y la acentuación de conflictos entre usuarios; y tercero, la implicación y participación del conjunto de la sociedad.

\section{CONCLUSIONES}

La gestión del regadío debe concebirse desde la compatibilidad dado que la tendencia actual prima la integración de intereses de carácter socioeconómico, ambiental, lúdico, patrimonial y territorial. Sabemos de la distinción entre usos consuntivos y no consuntivos del agua, pero resulta difícil compaginar sus respectivas necesidades y dinámicas. ¿Podemos seguir priorizando el regadío por detrás del consumo de boca sin condicionar con ello la dinámica ambiental del agua ni agravar el conflicto de intereses? La teoría presupone que los requerimientos ambientales no forman parte de los recursos hídricos que se ponen a disposición de los usos consuntivos, pero su gestión va entrelazada, de manera que todo episodio de eficiencia escasa o de contaminación supone desdibujar los parámetros de partida aceptados inicialmente. Sabemos que la escasez de recursos hídricos es subjetiva dado que viene determinada no solo por los condicionantes y las limitaciones físicas propias del recurso, sino también por las prioridades, las tradiciones y las políticas de gestión del mismo. Prueba de ello la encontramos en la dinámica del regadío catalán: la afinidad de discursos, la contraposición de ideales, la capacidad de adaptarse a cambios constantes o la priorización de un determinado modelo de desarrollo pueden resultar factores tanto o más providenciales que la propia disponibilidad física del recurso agua.

El agua y el suelo son cada vez más los principales motivos de preocupación de buena parte de la sociedad. Su disponibilidad en cantidad y calidad afianza la necesidad de legitimar sus usos y las consecuencias de las decisiones sobre los mismos. Es precisamente en la gestión de los recursos hídricos donde más se ha avanzado en un cambio de paradigma social focalizado en la complejidad. El paso de la Vieja cultura del agua -basada en el paradigma hidráulico como motor de desarrollo- a la Nueva cultura del agua ha simbolizado esta evolución en la concepción del entorno y sus recursos. Sus postulados, "los ríos son ecosistemas vivos", "no hay gestión del agua sin gestión del territorio" y "debate público y participación real en las decisiones" conforman los pilares de una nueva forma de interpretar las necesidades de las sociedades y las posibilidades del entorno a darles respuesta. En este contexto, la promoción de la gobernanza en el regadío en ámbitos como el catalán, con yuxtaposición de contextos en competencia, debe ser prioritaria para lograr su propia legitimación espacial y temporal.

\section{BIBLIOGRAFÍA}

AGÈNCIA CATALANA DE L'AigUA (2009): Pla de gestió del districte de conca fluvial de Catalunya, ACA, Barcelona (documento íntegro en la página web de la Agencia Catalana del Agua).

ALDOMÀ, I. (2012): La batalla per l'aigua. Una proposta per superar els desconcerts hídrics i garantir la gestió integral del seu cicle a Catalunya. Pagès Editors. Lleida, 198 p.

ALLAN, A. y WOUTERS, P. (2004): "What role for water law in the emerging "good governance" debate?", en Journal of Water Law, vol. 15, n 3-4, pp. 85-88.

BARTOLINI, F. et al. (2007): "The impact of water and agriculture policy scenarios on irrigated farming systems in Italy: An analysis based on farm level multi-attribute linear programming models", en Agricultural Systems, no 93, pp. 90-114.

BERBEL, J. et al. (2009): "Estimating demand for irrigation water in European Mediterranean countries through MCDM models", en Water Policy, vol. 11, n 3, pp. 348-361.

BERGER, C. y ROQUES, J. L. (2005): L'eau comme fait social. Transparence et opacité dans la gestion locale de l'eau. L'Harmattan. Paris, $185 \mathrm{p}$.

BREBBIA, C. A. et al. (2010): Sustainable Irrigation Management, Technologies and Policies III. WIT Press. Southampton, $272 \mathrm{p}$. 
BRUNSTAD, R. J. et al. (2005): "Multifunctionality of agriculture: an inquiry into the complementarity between landscape preservation and food security", en European Review of Agricultural Economics,

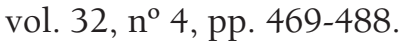

BURTON, M. A. (2010): Irrigation management. Principles and Practices. CABI. Cambridge, 375 p.

CONSELL ASSESSOR PER AL DESENVOLUPAMENT SOSTENIBLE (2010): Informe sobre el Pla de regadius de Catalunya (7/2010 de 12 de juliol), Barcelona: CADS.

COOK, C. y BAKKER, K. (2012): "Water security: Debating an emerging paradigm", en Global Environmental Change, $\mathrm{n}^{\circ} 22$, pp. 94-102.

DAVIES, E. G. R. y SIMONEVIC, S. P. (2011): "Global water resources modeling with an integrated model of the social-economic-environmental system", en Advances in Water Resources, n 34, pp. 684-700.

DORE, J. et al. (2012): "A framework for analysing transboundary water governance complexes, illustrated in the Mekong Region", Journal of Hydrology, n 466-467, pp. 23-36.

ERTSEN, M. (2006): "Colonial irrigation: Myths of emptiness", en Landscape Research, vol. 31, n 2, pp. 146-167.

GARRABOU, R. I RAMON-MUÑOZ, J-M. (2011): Aigua, agricultura i regadiu a la Catalunya contemporània, 1800-2010, UHE Working Paper 2011_15, Cerdanyola del Vallès: Unitat d'Història Econòmica (UAB).

GROENFELDT, D. (2006): "Multifunctionality of agricultural water: looking beyond food production and ecosystem services", en Irrigation and Drainage, n 55, pp. 73-83.

IGREMAP, S.L.P. (2010): Pla de Regadius de Catalunya 2008-20, Departament d'Agricultura, Alimentació i Acció Rural (Generalitat de Catalunya) y REGSA, Barcelona.

KAIKA, M. (2006): "Dams as symbols of modernization: The urbanization of nature between geographical imagination and materiality", en Annals of the Association of American Geographers, vol. 96, n 2, pp. 276-301.

KNOX, J. W. et al. (2012): "Water regulation, crop production, and agricultural water management: Understanding farmer perspectives on irrigation efficiency", en Agricultural Water Management, n 108, pp. 3-8.

LECINA, S. et al. (2010): "Irrigation modernization in Spain: Effects on water quantity and quality. A conceptual approach", en International Journal of Water Resources Development, vol. 26, n², pp. $265-282$.

LOPEZ-GUNN, E. (2009): “Agua para todos: The new regionalist hydraulic paradigm in Spain”, in Water Alternatives, vol. 2, n 3, pp. 370-394.

MUÑIZ, S. (2010): Agricultura i natura? Anatomia d'un conflicte. Claus i perspectives de futur del Canal Segarra-Garrigues. Diputació de Lleida, Societat Catalana d'Ordenació del Territori, Centre Tecnològic Forestal de Catalunya i Institut d'Estudis Ilerdencs. Lleida, 250 p.

MUÑIZ, S. (2009): "Disputas por el agua: ¿oportunidades para el desarrollo rural? Una investigación participativa con el transfondo del proyecto del Canal Segarra-Garrigues (Catalunya)", en Documentación Social, $\mathrm{n}^{\circ}$ 155, pp. 57-72.

OCA, J. (2009): "El regadiu a Catalunya", en Nota d’Economia, n 93-94, pp. 53-66.

ORTEGA SANTOS, A. (2012): "De aguas, tierras y políticas hidráulicas en la España contemporánea”, en Vínculos de Historia, $\mathrm{n}^{\circ}$ 1, pp. 73-94.

ÖZEROL, G. et al. (2012): "Irrigated agriculture and environmental sustainability: an alignment perspective", en Environmental Science E Policy, n² 23, pp. 57-67.

PAHL-WOSTL, C. (2010): "Water governance in times of change", en Environmental Science E Policy, $\mathrm{n}^{\mathrm{o}} 13$, pp. 567-570.

PARDO, M. (1999): "El impacto social (positivo y negativo) de las construcciones hidráulicas", en ARROJO AGUDO, P. y MARTÍNEZ GIL, F. J. (Coords.): El agua a debate desde la Universidad. Hacia una Nueva Cultura del Agua. I Congreso Ibérico sobre Gestión y Planificación de Aguas. Zaragoza, 14-18 de septiembre de 1998, pp. 1-8. 
PAVÓN, D. (2008): Gran obra hidràulica i territori a les conques de la Muga i del Fluvià (1850-1980). Institut d'Estudis Empordanesos (Figueres); Patronat Francesc Eiximenis i Universitat de Girona (Girona).

PERVANCHON, F. y BLOUET, A. (2002): "Lexique des qualificatifs de l'agriculture", en Le Courrier de l'environnement de l'INRA, n 45, pp. 117-137.

PRITCHARD, S. B. (2004): "Reconstructing the Rhône: The cultural politics of nature and nation in contemporary France, 1945-1997", en French Historical Studies, vol. 27, no 4, pp. 765-799.

RIBAS, A., et al. (2012): "Perception et valoration sociale de l'irrigation traditionnelle dans le Bas Ter (Bas Ampurdan, Catalogne)”, en ASPE, C. (COORD.): De l'eau agricole à l'eau environnmentale. Résistance et adaptation aux nouveaux enjeux de partage de l'eau en Méditerranée. Éditions Quae, París, pp. 199-208.

RICART, S. y ROCA, A. (2013): "Entre l'agriculture et l'environnement: l'écologisation de la Politique Agricole Commune (PAC) comme légitimation des exigences sociales", en Colloque international interdisciplinaire Dynamiques environnementales, politiques publiques et pratiques sociales: quelles intéractions? Université de Toulouse Le Mirail. Toulouse, 4-7 de junio.

RICART, S. et al. (2011): “¿Hacia una gestión territorial del regadío ?: El Canal Segarra-Garrigues (Lérida, Cataluña)", en Colloque Usages écologiques, économiques et sociaux de l'eau agricole en Méditerranée: quels enjeux pour quels services?. LPED-IMEP, Université de Provence. Marsella, 20-21 de enero.

SWYNGEDOUW, E. (1999): "Modernity and Hybridity: Nature, regeneracionismo, and the production of the Spanish waterscape", en Annals of the Association of American Geographers, vol. 89, n 3, pp. 443-465.

TROPP, H. (2007): "Water governance: trends and needs for new capacity development", en Water Policy, no 9, pp. 19-30.

TURRAL, H. et al. (2010): "Investing in irrigation: Reviewing the past and looking the future", en Agricultural Water Management, nº 97, pp. 551-560.

VENTURA, M. (2004): "Conflictes socioterritorials i participació pública en la gestió de l'aigua de la conca del riu Muga (Alt Empordà)”. (Tesis doctoral). Girona: Institut de Medi Ambient, Universitat de Girona.

WIEK, A. y LARSON, K. L. (2012): "Water, people, and sustainability: A systems framework for analyzing and assessing water governance regimes", en Water Resources Management, n 26, pp. 3153-3171. 